\title{
EVALUACIÓN DIDÁCTICA DE SOFTWARE EDUCATIVO
}

Sara Galbán Lozano Claudia F. Ortega Barba

\section{RESUMEN}

Al ser el campo educativo un ámbito del actuar humano, también se ha visto -y se ve- influenciado por los cambios radicales del mundo. Se le imponen, así, nuevos retos a los cuales responder: la educación a lo largo de toda la vida, la cooperación internacional, la educación multicultural, el manejo de nuevas tecnologías, la revisión constante de los planes y programas de estudio, la evaluación continua de los procesos educativos... Es en este contexto donde se presenta el gran reto de evaluar no sólo los procesos sino los productos creados desde la relación educación-tecnología.

En un principio, la tecnología se relacionó sólo con procesos productivos y bélicos; hoy, sin embargo, los adelantos superan estos ámbitos, desbordándose a muchos otros sectores, entre ellos, el de la educación.

Tradicionalmente, los procesos educativos formales se han realizado de manera presencial, siendo uno de los recursos predominantes, el libro. Aunque los libros no desaparecerán, no podemos cerrar los ojos frente a los avances que la tecnología provee; es así como se incorporan las herramientas digitales como medios educativos: CD-ROM, DVD y las redes que propician novedosas técnicas de educación no presencial.

Lo anterior obliga a los profesores y especialistas en el ámbito de la educación a utilizar estas nuevas herramientas, de manera que la exigencia para el profesor no se reduce ya al mero conocimiento de los libros y materiales impresos de cada 
especialidad, sino que ahora, además de ese conocimiento, requiere la utilización de las novedades tecnológicas.

Así como existen lineamientos para evaluar si un libro cumple o no con los objetivos estipulados en los programas, y si existe coherencia lógica y psicológica entre el material y el destinatario, de la misma manera los materiales digitales deberán evaluarse. Sin embargo, la vertiginosidad del avance tecnológico y la concreción de ésta en diversos tipos de software, ha desbordado el fenómeno de creación, superando su evaluación.

En un primer momento esto podría considerarse una deficiencia; no obstante, se presenta más bien como una oportunidad para profundizar en el campo de la evaluación. De esta reflexión se desprende la siguiente propuesta enca-minada a la evaluación de software educativo.

\section{INTRODUCCIÓN}

En general, el software puede definirse como el conjunto de programas que permiten a una persona utilizar la computadora para diferentes fines; en este caso, nos interesan aquellos componentes operativos facilitadores del proceso educativo.

Específicamente el software educativo se ha dirigido al desarrollo de habilidades mentales y creación de contenidos. "El software para el desarrollo de habilidades mentales trabaja en combinación con recursos multimedia y tiene como principal objetivo poner en práctica operaciones de pensamiento convergente y divergente, lineal y lateral. Las funciones principales de este tipo de programas son la ejercitación y la práctica, éstas muchas veces se evalúan de manera automatizada" ${ }^{1}$.

${ }^{1}$ ORTEGA, C., "Educación y tecnología: el software como apoyo al proceso de aprendizaje", en Revista Panamericana de Pedagogía: saberes y quehaceres del pedagogo., p.192. 
El software de contenido puede considerarse como un libro electrónico auxiliado de la posibilidad multimedia y de la interactividad; su función es la presentación y trasmisión de información.

\section{¿QUÉ ES EVALUAR?}

El ámbito de la instrumentación didáctica se compone de cuatro momentos: diagnóstico de necesidades; planeación; realización; evaluación.

De ellos, la evaluación es el componente más olvidado, entre otras cosas por la dificultad que implica su definición, pues muchas veces se le confunde con el término de medición.

Es pues necesario determinar que la estrategia propuesta en este trabajo se refiere a la evaluación como un proceso integral; y por ello consideramos necesario establecer un marco conceptual acerca de la misma.

Por evaluación entendemos el establecer un juicio o valor sobre algo. No es sinónimo ni debe confundirse con la medición, pues este término se refiere al proceso de colección de datos, la mayoría de las veces cuantitativos, los cuales luego serán utilizados como base para establecer los juicios. Por lo tanto, el concepto evaluación abarca más que el de medición, pues al medir no necesariamente se está evaluando. Pero para realizar una adecuada evaluación se necesita que el proceso de medición sea válido y confiable.

La evaluación es el esfuerzo continuo que integra tanto los efectos del proceso de enseñanza aprendizaje, como el contexto en el cual se desarrolla, para cumplir los propósitos previamente definidos ${ }^{2}$.

Para cumplir los propósitos de la evaluación se consideran en el proceso tres facetas: la evaluación educativa, la evaluación instruccional y la evaluación curricular.

${ }^{2}$ ORTÍZ, A., Diseño y evaluación curricular., p.357. 
La evaluación educativa se realiza en el ámbito de la escuela y engloba los procesos académicos y administrativos en los que se desarrolla el proceso formativo.

La evaluación instruccional comprende la valoración de los logros de los estudiantes, de la ejecución del maestro y de la efectividad de las metodologías y materiales utilizados.

La evaluación curricular incluye las dos anteriores. Es precisamente de ella de donde se desprende la presente propuesta, pues el software educativo puede ser un material utilizado en el proceso de enseñanza aprendizaje.

\section{¿EL PORQUé de LA EVAlUACIÓN?}

Muchas veces nos preguntamos por qué es necesario evaluar un material digital. La respuesta no es privativa de los materiales digitales, la cultura de la evaluación marca la pauta para la toma de decisiones aun en los ámbitos de la vida cotidiana. Ejemplo de ello es que, al momento de comprar una televisión, un DVD o una computadora, por citar algunos casos, diagnosticamos las ventajas y desventajas que nos ofrecen.

Esa misma actitud que en lo cotidiano es tan natural, resulta indispensable en el ámbito escolar. Algunas ventajas que nos reporta esta conducta, que ha de convertirse en un hábito, se mencionan a continuación:

1. Evaluar conlleva la manipulación del objeto en cuestión, y por ende, el acercamiento a su forma de operar; la evaluación es un medio idóneo para lograr un primer contacto con las herramientas tecnológicas.

2. Conocer la variedad de materiales digitales que el mercado ofrece; la evaluación implica, necesariamente, la comparación.

3. Al determinar las ventajas y desventajas de los materiales existentes, puede participarse en la creación de medios expresamente diseñados para cada asignatura y, con ello, lograr un mayor aprovechamiento de la materia. Cabe mencionar que el crear un software educativo implica 
forzosamente el manejo de técnicas informáticas, las cuales sólo poseen los especialistas. Sin embargo, el docente es pieza clave para la elaboración de los contenidos, pues domina la materia y las características del nivel educativo donde se inscribe.

4. La evaluación de materiales permite manejarlos con profesionalidad y profundidad, lo cual favorece aprendizajes significativos en los alumnos y en el mismo profesor.

\section{EL CONTEXTO DE LA EVALUACIÓN}

Los materiales digitales no se encuentran aislados, forman parte de un entramado de elementos que conforman el proceso educativo. Por ello resultaría estéril fundamentar la evaluación en el mero material didáctico.

Así como la instrumentación didáctica está conformada por cuatro momentos, ya mencionados, también se compone de siete elementos:

- Educando-educador.

- Objetivo educativo.

- Contenido educativo.

- Metodología o estrategia didáctica (incluye métodos, técnicas y procedimientos).

- Recursos didácticos.

- Tiempo.

- Lugar.

En el contexto de la evaluación, el recurso didáctico debe vincularse con los demás elementos para ser evaluado. Así, surge nuestra propuesta que comprenderá dos vías: la relacional o interna, y la externa.

\section{EVALUACIÓN RELACIONAL}

Esta evaluación se basa en el análisis del software educativo como recurso didáctico y su relación con los demás elementos del proceso educativo. 


\section{A) Relación del Software Educativo con el Educando}

Desde una perspectiva eminentemente constructivista, se habla de la significatividad psicológica que no es otra cosa que la adecuación entre el contenido, materiales, estrategias y conformación biopsicosocial del educando. Es así como el software educativo deberá ir en consonancia con el desarrollo biopsicosocial del destinatario. Es decir, tendrá que responder a las características de la etapa evolutiva, estructura mental, los conocimientos, habilidades y actitudes desarrolladas; así como al contexto sociocultural y estilo de aprendizaje del educando.

En cuanto a los estilos de aprendizaje, cabe resaltar que la maleabilidad del software educativo permite diversificar los procesos de aprendizaje y, por ende, atender a las diferentes formas de aprender aun cuando un aula sea muy numerosa.

\section{B) Relación del Software Educativo con el Educador}

Éste es un rubro al cual se le empieza a dar importancia a partir de una práctica educativa más especializada; con ésta se ha determinado la necesidad de que los materiales de instrucción vayan en consonancia con la persona del educador. En ello debe tomarse en cuenta la personalidad, estructura mental y estilo de enseñanza de cada profesor.

\section{C) Relación del Software Educativo con el Objetivo Educativo}

El sentido relacional de estos elementos abarca dos vertientes, por un lado, la necesidad de que el contenido del software se inserte dentro de los objetivos generales del currículo, y por el otro, al utilizar éste es indispensable establecer el para qué de su uso.

Es importante destacar que el software no debe utilizarse como pretexto para ocupar el tiempo destinado a la sesión; de ser así, más que benéfico, podría ocasionar descontento y descontrol entre los alumnos. 


\section{D) Relación del Software Educativo con el Contenido}

Esta relación conduce a dos planteamientos: por un lado, que el contenido esté en consonancia con el material trabajado en la asignatura, y por el otro, que la representación de los contenidos se proporcione de una manera lógica.

En la práctica educativa se habla del aprendizaje de diversos contenidos curriculares: los contenidos declarativos (hacen referencia al conocimiento), los contenidos procedimentales (a las habilidades) y los contenidos actitudinales (a las actitudes) ${ }^{3}$. Es así como, el software educativo está integrado por diversos tipos de contenidos que, además, van en consonancia con el para qué fueron hechos.

Consideramos que el software educativo puede, eminentemente, desarrollar contenidos declarativos y procedimentales, esto es, conocimientos y habilidades, aunque sin descartar las habilidades de pensamiento.

\section{E) Relación del Software Educativo con la Metodología o Estrategias Didácticas}

En esta relación existen tres variables. Por un lado, el que la utilización del software educativo sea catalogada como una estrategia de enseñanza aprendizaje, y por tanto ha de adecuarse al educando, al educador, a los objetivos y a los contenidos. Por el otro, la forma en cómo se utilizará el propio software, pues su manejo interno requiere de estrategias de navegación para optimizar su uso, mismas que se adquieren con la práctica. Una tercera variable se determina por las actividades que el mismo software provee y que sirven como reforzadores del aprendizaje.

${ }^{3}$ Cfr. DÍAZ BARRIGA, F. y HERNÁNDEZ ROJAS, G., Estrategias docentes para un aprendizaje significativo: una interpretación constructivista., p.29-32. 


\section{F) Relación del Software Educativo con los Recursos Didácticos}

Ya se ha dicho que el software educativo guarda relación con el recurso didáctico; para su eficaz utilización depende mínimamente de ciertos medios como son: luz eléctrica, una computadora con el sistema operativo adecuado y un profesor capacitado para su uso.

\section{G) Relación del Software Educativo con el Tiempo}

Como este medio no tiene una temporalidad lineal, puede ocuparse según las indicaciones del profesor o las del creador del mismo.

Es por eso que el tiempo no es un punto relevante para la evaluación.

\section{H) Relación del Software Educativo con el Lugar}

Tiene relación, específicamente, con el lugar de creación y el lugar de utilización, puesto que no pueden adoptarse productos sino adaptarlos, usándolos de acuerdo a las características del contexto y del mismo alumno.

\section{EVALUACIÓN EXTERNA}

En la evaluación externa se miden los aspectos técnicos, estéticos y económicos del software educativo, tomando en cuenta lo siguiente:

- Calidad del entorno audiovisual.

- Grado de "amigabilidad" de la interfase ${ }^{4}$.

- Grado de interactividad.

- Facilidad de navegación.

${ }^{4}$ Se entiende por interfase, el conjunto de programas que controlan la forma visual en que el sistema informático se presenta al usuario. 


\section{CONSIDERACIONES FINALES}

A partir de la propuesta presentada sobre los lineamientos generales para evaluar el software educativo, hemos de aclarar que se trata de una propuesta flexible y, por ende, ha de adaptarse tanto al proceso educativo específico, como a las características propias de cada material.

También, que la didáctica -aun con todos los adelantos tecnológicos y las modalidades que esto implica para el proceso de enseñanza aprendizaje-, sigue y seguirá teniendo vigencia, puesto que constituye el fundamento para la optimización de los procesos de enseñanza aprendizaje en la vida del aula, entendiendo esto desde una perspectiva abierta, donde el concepto de aula no se reduce al ámbito escolar, sino a cualquier espacio educativo. 
DÍAZ BARRIGA, F. y HERNÁNDEZ ROJAS, G., Estrategias docentes para un aprendizaje significativo: una interpretación constructivista., México., McGraw Hill., 1998., 232 p.

ORTEGA, C., "Tecnología y educación: el software como apoyo al proceso de aprendizaje”., Revista Panamericana de Pedagogía: saberes y quehaceres del pedagogo., Revista semestral., No.3., Nueva Época., 2002., Universidad Panamericana., Facultad de Pedagogía., México., pp. 189-196.

ORTIZ, A., Diseño y evaluación curricular., República Dominicana., Edil., 1997., 613 p. 